\title{
The definition of scale series for atlas maps depending on the density of mapped objects
}

\author{
Zagrebin G.I. ${ }^{\text {a, }}$, Krylov S.A. ${ }^{\text {b }}$ \\ ${ }^{a}$ Moscow State University of Geodesy and Cartography (MIIGAiK), gleb@cartlab.ru, \\ ${ }^{b}$ Moscow State University of Geodesy and Cartography (MIIGAiK), krylov@cartlab.ru \\ * Corresponding author
}

Keywords: Atlas, map scale, automatization, density of objects

\begin{abstract}
:
The definition of scale series i.e. the identification of groups of scales in which maps will be created is one of the important tasks of designing the mathematical basis of the atlas. Map scale calculation based on the map format and the size of the mapping area is the most common approach to calculating the scale of the being created atlas maps. This approach allows to take into account the margins and layout of maps on the page. But usually these parameters aren't known even at the design stage of a new atlas. So it is also necessary to take into account the completeness and detail of the content of the atlas maps at the stages of designing the atlas and determining the scale series for small-scale maps. The authors propose to carry out the calculation of scale series for atlas maps depending on the density of the mapped objects and the graphical load associated with it. In this case, both the density of general geographical objects (for example, settlements) and the density of objects of a thematic content (for example, volcanoes and earthquake epicentres, industrial centres, etc.) can be taken into account. These factors are important when designing general geographic atlases and when creating atlases containing a large number of thematic maps respectively. The method to determine the scale series for atlas maps depending on the density of the mapped objects is developed by authors and presented in Figure 1.

The initial stage of the method is the determining of the density of the mapped objects for a given region. It will be basis for map scale calculation. In case of creating thematic maps, the density of objects depends on the selected indicator and the cartographic image method used, so the density is calculated in different ways. For example, when displaying thematic content with sign icons localized in settlements (for example, health facilities), it is necessary to determine not the total number of thematic objects, but the number of settlements in which this theme will be displayed. To address the issue of inset map, it is also necessary to know the number of objects in a particular locality. Also the total length of the road network is determined when using linear signs on a map, for example, to show passenger and cargo traffic.
\end{abstract}

The map scale is calculated for each territory to be mapped, taking into account the density of map objects, indicators of thematic maps and the size of conventional symbols according to the following formula:

$$
M_{K}=\sqrt{\frac{K_{N} S_{M}}{N}}
$$

where $\quad M_{K}=$ scale denominator expressed in km per $1 \mathrm{~mm}$

$K_{N}=$ coefficient of optimal graphical load

$S_{M}=$ area of mapped territory in sq.km

$N=$ total graphical load of the designed map in sq.mm.

Formula (1) is common. The total graphical load of the projected map is calculated differently depending on the type of localization of the mapped objects (linear, point), their thickness and sizes of conventional symbols.

As a result of the calculations, the scale series of atlas maps is being defined. This series makes it possible to assess the scales in which need to create maps for the complete display of thematic information. The obtained scales aren't multiple and usually their minimum and maximum values are located far from each other. It is necessary to determine the values outside the normal series for further processing and decide on the creation of large-scale inset maps and small-scale overview maps, or change the ways of displaying the corresponding indicators of thematic maps.

Next, it is necessary to reduce the number of scales in the defined series by classifying the scale series according to the Natural breaks classifications. At this stage, the question of choosing the number of classes is important, because this parameter will indicate the number of scales in the final scale series. After selecting the scale classes, we find the 
resulting scale for each class. The scale should be close to the average in the class, but at the same time be rounded and multiple to the scales of other classes. The obtained average scales will be the final scale series.

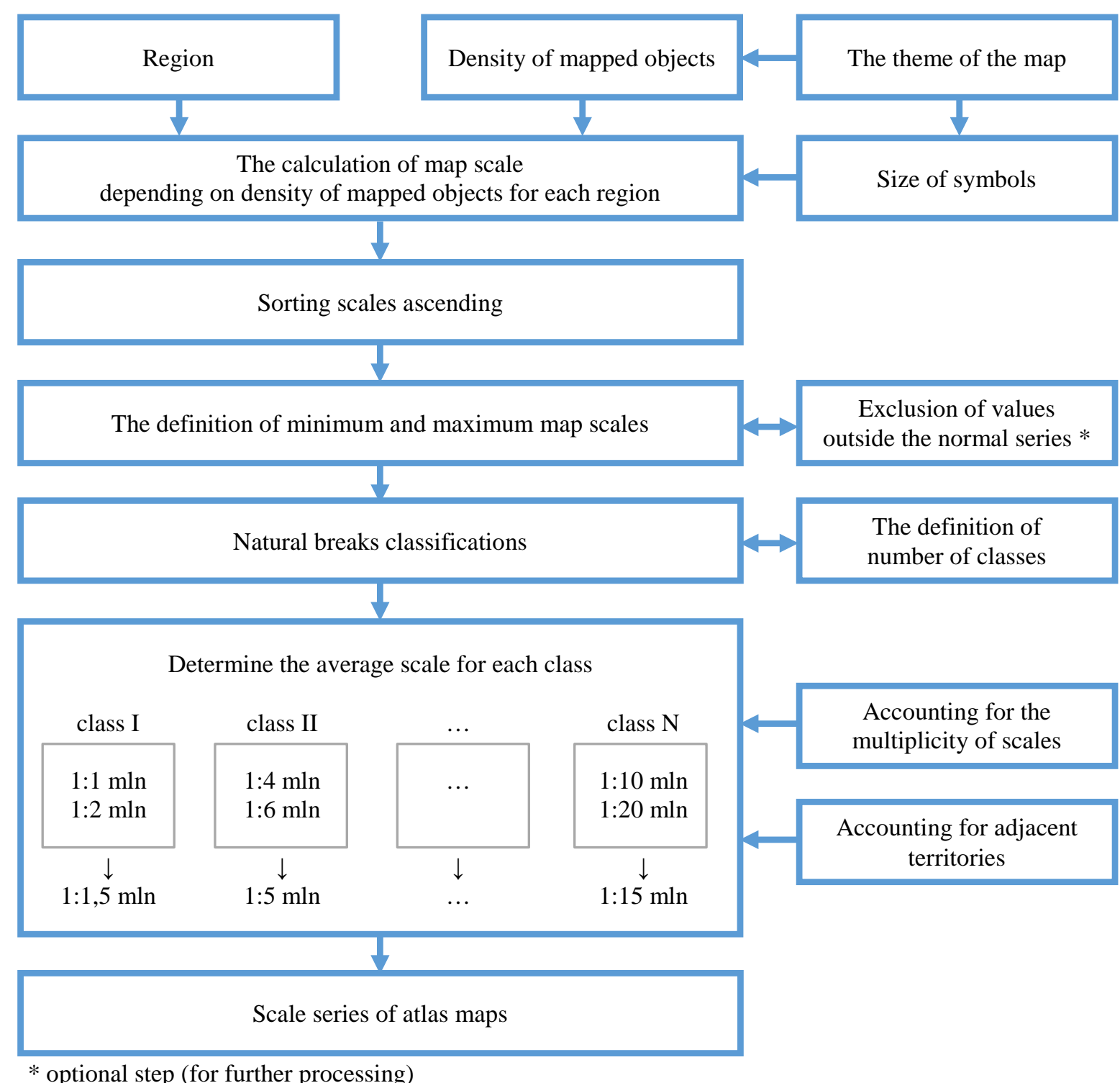

Figure 1. The method to determine the scale series for atlas maps depending on the density of the mapped.

The developed method allows defining the scale series of atlas maps in an automated mode, taking into account the thematic content of maps and the size of conventional symbols. Also it make possible to identify areas with a unique density of objects (industrial sites, reserves), to speed up the design of atlases.

\section{Acknowledgments:}

This work was supported by Ministry of education and science of Russian Federation, project 5.8029.2017/8.9. 